\title{
Die Anfänge des Klosters Murrhardt
}

\author{
von Gerhard Fritz
}

Die 2016/17 anstehenden Jahrestage zur 1200. Erstnennung mehrerer Orte rund um Murrhardt (Sulzbach, Fichtenberg, Kaisersbach) sind Anlass, sich mit den auf 816/817 datierten Murrhardter Quellen zu befassen, auf denen diese Jahrestage beruhen. In meinem 1982 veröffentlichen Buch „Kloster Murrhardt im Früh- und Hochmittelalter" ging es u.a. um die Gründung des Klosters Murrhardt. ${ }^{1}$ Heinrich Wagner hat 2001 das Thema wieder aufgegriffen. ${ }^{2}$ Er konnte durch die Neu-Edition bereits 1982 herausgegebener Quellen deren Lesung präzisieren und erkennt und identifiziert innerhalb dieser Quellen insbesondere eine neue, bislang nicht erkannte Urkunde Kaiser Ludwigs des Frommen von 816. Dabei kam er zu einer Reihe neuer Erkenntnisse. Im Zusammenhang damit stellte er Thesen auf, die zu diskutieren sein werden. Nicht zuletzt wird auch auf verschiedene örtlich-geographische Zuschreibungen Wagners einzugehen sein.

\section{Die gefälschten Urkunden von 788 und 817}

Eine Urkunde von 788 und eine von 817 erwähnen das Kloster. 788 schenkt Karl der Große, so heißt es in der älteren Urkunde, die ,,in seinem Eigentum befindlichen Orte Neustadt und Homburg“, beide am Main, (loca quedam sibi proprietate, Nuwenstat scilicet, Hohenburg) und „die andern kleinen Zellen“ Amorbach, Schlüchtern und Murrhardt (cellulasque alias Amerbach, Sluchtern et Murrahart) an den Würzburger Bischofstuhl (ad Wirtzeburgensis sedem civitatis). Außerdem wird in der Urkunde Bezug genommen auf eine weitere, nicht erhaltene Urkunde von König Pippin, dem Vater Karls des Großen, der die Orte bereits an den ersten Würzburger Bischof Burkhard übertragen habe. ${ }^{3}$

817 nimmt Karls Sohn, Kaiser Ludwig der Fromme, das von ihm gestiftete Kloster Murrhardt in seinen Schutz. ${ }^{4}$ Der ehrwürdige Einsiedler Walterich (venerabilis heremita, Walthricus nomine) sei vor den Kaiser getreten (maiestatis nostrae adiens presentiam) mit der Bitte, der Kaiser möge ihm den Ort schenken, an

1 Gerhard Fritz: Kloster Murrhardt im Früh- und Hochmittelalter. Eine Abtei und der Adel an Murr und Kocher. (FWFr 18). Sigmaringen 1982.

2 Heinrich Wagner: Die Privilegierung des Klosters Murrhardt durch Ludwig den Frommen. In: Deutsches Archiv für Erforschung des Mittelalters 57 (2001), S. 421-450.

3 WUB 1, Nr. 35, S. $36 \mathrm{f}$.

4 WUB 1, Nr. 78, S. $87 \mathrm{ff}$. 
dem er bisher Gott in der Einsamkeit angebetet habe (ut locum sibi, ubi secretius deo vacaret, largiri dignaremur). Daraufhin habe der Kaiser diesem bewundernswürdigen Mann erlaubt, im Wald nahe seiner Burg namens Hunnenburg nahe dem Ufer der Murr zu leben (ipsum venerabilem virum [...] in nemore prope castrum nostre proprietatis, quod vulgo Hunemburg dicitur, iuxta rivum Murra vocatur, habitare permisimus), ja er habe diesen lobenswerten Mann sogar als seinen Beichtvater ausgewählt (laudabilem vero viri [...] in peculiarem nobis confessorem elegimus). Wenig später seien zwölf Brüder hinzugekommen, und der Kaiser habe angeordnet, dass diese gemäß der Regel des heiligen Benedikt dort das mönchische Leben zu führen hätten (non multo post solitariam eius vitam necessario mutantes, duodecim fratres [...] secundum instituta regule beati Benedicti monasticam vitam ducere debent, instituimus). Die Gründung dieses Klosters wird mit zahlreichen Einzelheiten näher beschrieben. Auf einige dieser Einzelheiten wird nachfolgend genauer einzugehen sein.

Das Problem ist, dass beide Urkunden nicht im Original erhalten sind, sondern nur als Fälschungen bzw. Überarbeitungen. Auf diesen Charakter der beiden Urkunden habe ich 1982 wiederholt und ausdrücklich hingewiesen.

Die näheren Umstände der Urkunde von 788 sind nur vor dem Hintergrund des späten 10. Jahrhunderts verständlich. Damals - konkret im Jahr 993 - gelang es dem Bistum Würzburg, den Besitz von insgesamt fünf Klöstern von Kaiser Otto III. bestätigt zu bekommen. Es handelt sich um dieselben Klöster bzw. Orte, die in der Urkunde von 788 genannt werden, also Neustadt, Homburg, Amorbach, Schlüchtern und Murrhardt. Heinrich Wagner, der sich 2001 intensiv mit den Murrhardt betreffenden Urkunden des späten 8. und frühen 9. Jahrhunderts auseinandergesetzt hat, spricht der Urkunde von 788 jeglichen Wahrheitsgehalt ab. Er sieht sie als reine Würzburger Fälschung, mit der der Bischof von Würzburg den Kaiser überzeugen wollte, die fünf Klöster bzw. Orte an das Bistum zu übertragen, was dann auch 993 geschah. ${ }^{5}$ Die gesamte ältere Forschung, die eine - wenn auch überarbeitete bzw. verfälschte - echte Vorlage für den heute überlieferten Wortlaut der Urkunde von 788 annahm oder zumindest als möglich erachtete, erklärt Wagner ohne weitere Begründung als hinfällig. ${ }^{6}$ Auf verschiedene Indizien einschließlich archäologischer Befunde, ${ }^{7}$ die auf ein Engage-

5 Wagner 2001 (wie Anm. 2), S. 421 f. Vgl. auch der von Wagner 2016 für die Gemeinde Fichtenberg verfasste und für eine dortige Jubiläumspublikation vorgesehene Text: Fichtenberg vor dem Übergang an Limpurg 1338. In: 1200 Jahre Fichtenberg. Jubiläumsband. Hg. von der Gemeinde Fichtenberg. Red. Christoph Bittel, S. 57-66, in dem Wagner aber nur seine Aussagen von 2001 paraphrasiert.

6 Vgl. die Zusammenstellung der älteren Literatur bei Fritz 1982 (wie Anm. 1), S. 40, außerdem auch im Anhang zur Online-Edition des WUB 1, Nr. 35, S. 36 f.

7 Vgl. dazu: Fritz 1982 (wie Anm. 1), S. 44, zu archäologischen Befunden auch außerhalb der Klosterkirche. Ulrike Plate: Das ehemalige Benediktinerkloster St. Januarius in Murrhardt. Archäologie und Baugeschichte. Stuttgart 1996, S. 29ff und 105, ordnet eine ganz frühe Holzbauphase (A1) und eine darauf folgende Steinbauphase (A2) im Bereich von Vierung und Querschiff der heutigen Murrhardter Klosterkirche als römisch ein. 
ment der Sippe des Würzburger Bischofs Megingoz zur Regierungszeit des Königs Pippin zwischen etwa 753 und 768 in Murrhardt hinweisen, ${ }^{8}$ geht Wagner nicht ein. Die Sippe des Megingoz dürfte mit der Sippe des späteren Murrhardter Klostergründers Walterich verflochten gewesen sein. Gut gekannt haben sich Walterich und Megingoz, wie noch zu zeigen sein wird, auf jeden Fall. Von mir seinerzeit als denkbar angenommene, aber auf jeden Fall gescheiterte Klostergründungsversuche in dieser Zeit (oder sogar noch vorher?) bestreitet Wagner.

\section{Klösterliche Anfänge schon im 8. Jahrhundert?}

Nun ist in der gefälschten Urkunde von 788 nirgends die Rede von einem Kloster Murrhardt, sondern von einer cellula Murrahart (genauso wie auch Amorbach und Schlüchtern als cellulae) bezeichnet werden. Cellula ist die Verkleinerungsform von cella, also Zelle, und als solche werden (Mönchs-)Zellen bezeichnet, kleine pseudoklösterliche Niederlassungen, die noch nicht die Dimension eines regulären Klosters erreicht haben. Eine geistliche Niederlassung hat es in Murrhardt zu dieser Zeit nach Ausweis der archäologischen Funde aber mit Sicherheit gegeben: Entweder in Form der (Marien-)Kirche, der heutigen Walterichskirche auf dem Berg oberhalb des späteren Klosters oder vielleicht schon unten im Tal im Bereich des späteren Klosters. Zwar liefert die Publikation von Ulrike Plate über die 1973 durchgeführten archäologischen Ausgrabungen des Klosterbereichs keinen entsprechenden Hinweis, ${ }^{9}$ der Ausgräber von 1973, Rolf Schweizer, interpretiert die damaligen Grabungsbefunde jedoch völlig anders. Er sieht insbesondere am Ostende der karolingischen Klosterkirche eine Befundsituation, die in die nachrömische Zeit, aber in die Zeit vor der Klostergründung des Walterich verweist. Dort befand sich nach Schweizer ein großes steinernes Gebäude, von dem bei den Grabungen von 1973 nur die westliche Außenmauer erfasst sei (vgl. Abb. 3). Plate sieht diese Mauer dagegen als östliche Abschlussmauer einer mutmaßlichen Erweiterung der Klosterkirche des 9. Jahrhunderts. Mit Schweizers Deutung müssen auch mehrere Gräber westlich dieser Mauer neu gesehen werden: Sie wären demnach Außenbestattungen des offenkundig für religiöse Zwecke genutzten Gebäudes. Dieses Gebäude wäre zusammen mit einigen von Plate nicht erwähnten Hinweisen auf ein Holzgebäude im Nordbereich der heutigen Klosterkirche - demnach als Kloster bzw. Klostergründungsversuch vor Walterich zu deuten. ${ }^{10}$ Näheres dazu ergibt sich aus den beigefügten, freundlicherweise von Rolf Schweizer zur Verfügung gestellten

8 Fritz 1982 (wie Anm. 1), S. 42-49.

9 Plate (wie Anm. 7), S. 29-41.

10 Vgl. dazu auch Rolf Schweizer: St. Walterich und sein Kloster in Murrhardt. Sein Leben und Wirken. Horb 2013, S. 28-43. Ein Foto eines der nach Schweizer westlich der fraglichen Mauer 1973 angeschnittenen Gräber bei Plate (wie Anm. 7), S. 35, Abb. 21. 
Grundrisszeichungen aufgrund der Ausgrabungsbefunde von 1973. Schweizers Zeichnungen unterscheiden sich erheblich von denen Plates.

Reine Phantasie ist also die cellula-Aussage der Urkunde von 788 auf keinen Fall. Auch auf die Argumentation der aus später Überlieferung - nämlich der Reichenauer Chronik des Gallus Öhem (um 1445-1522, Entstehungszeit der Chronik Ende des 15. Jahrhunderts) - stammenden Nachricht, dass Pirmin (um 670-753) neben anderen Orten auch in Moratt in Wirtzburger bistům als Gründer tätig war, ${ }^{11}$ geht Wagner nicht weiter ein. Öhem sagt nicht explizit aus, was Pirmin in Murrhardt gegründet haben soll: Eine Pfarrei? Eine Zelle? Ein Kloster? Auffällig ist aber, dass Pirmin an allen andern Orten, die Öhem im Zusammenhang mit Murrhardt aufzählt, Klostergründungen oder zumindest Gründungsversuche unternommen hat. Jedenfalls gab es für Öhem keinerlei Motiv, Pirmins Aktivitäten in Murrhardt zu erfinden. Da Öhem von den Urkunden von 788 und 817 keine Kenntnis hatte, muss er auf irgendeine, heute nicht mehr erhaltene Reichenauer Überlieferung zurückgegriffen haben.

Noch ein weiteres Indiz deutet christliche Aktivitäten in Murrhardt lange vor Walterich an. Im 1642 entstandenen Chronicon Murrhartense des Adam Adami findet sich eine irritierende Mitteilung. Adami war im Zuge der Rekatholisierung des Klosters Murrhardt dort während des Dreißigjährigen Krieges Prior und versuchte - u. a. durch Befragung alter Einwohner - die örtlichen katholischen Traditionen zu erforschen. Dabei erwähnt Adami einen stark fließenden, heilkräftigen Brunnen, der als $S$. Ruprechtesbronn bezeichnet werde und in einem Tal eine halbe Stunde von Murrhardt entfernt liege. Weshalb der Brunnen nach dem heiligen Rupert benannt war, konnte Adami nicht ermitteln. Er erwägt - offenbar ohne Kenntnis des Baiern-Apostels und in Analogie zur Walterichs-Überlieferung - auch, dass bei dem Brunnen ein heiliger Eremit Rupert gelebt haben könnte. Nun gibt es in Murrhardt zwar einerseits keinerlei weitere RupertsÜberlieferungen, andererseits gibt es auch keinerlei Grund, weshalb man sich noch lange nach der Reformation - einen solchen Bezug auf einen katholischen Heiligen einfach grundlos ausgedacht haben sollte. Der heilige Rupert (um 650718) ist zwar in erster Linie als Apostel der Baiern und erster Bischof von Salzburg bekannt, er stammte aber aus Worms. Auch dort war er Bischof, und dort starb er auch und wurde - bis zur Überführung seiner Gebeine nach Salzburg 774 - dort auch zunächst bestattet. ${ }^{12}$ Murrhardt läge auf der von Rupert offenbar

11 Vgl. das ausführliche Zitat aus Öhems Chronik bei Fritz 1982 (wie Anm. 1), S. 47. Zu Öhem jüngst Pia Eckhart: Ursprung und Gegenwart. Geschichtsschreibung in der Bischofsstadt und das Werk des Konstanzer Notars Beatus Widmer (1475-ca. 1533) (Veröffentlichungen der Kommission für geschichtliche Landeskunde in Baden-Württemberg, Reihe B, 207). Stuttgart 2016, S. 527-530. Öhems Biographie zeigt nirgends einen Bezug zu Murrhardt oder zum Bistum Würzburg. Er muss also im Zusammenhang mit Murrhardt tatsächlich auf Reichenauer Quellen zurückgegriffen haben. 12 Adamis Chronicon Murhartense befindet sich in der Universitätsbibliothek Würzburg M. ch. q. 85, der Hinweis auf dem S. Ruprechtsbrunnen auf f. 364r. Vgl. zu Rupert: Roman Deutinger: Rupert von Salzburg. In: NDB 22 (2005), S. 272 f. Der nach Adami in einem Tal eine halbe Stunde von Murrhardt gelegene, stark fließende Brunnen (media hora distans fons qui fortissime et largissime 
mehrfach begangenen Strecke von Worms nach Salzburg und anderen bairischen Orten durchaus auf dem Weg. Sollte es tatsächlich einen Bezug des heiligen Rupert nach Murrhardt geben, wären die christlichen Anfänge des Ortes noch über die Zeit Pirmins ins frühe 8. Jahrhundert, wenn nicht gar ins späte 7. Jahrhundert, zurückzuverlegen.

Wie auch immer: Es ist aufgrund der schriftlichen Quellen und der archäologischen Befunde (und eventuell auch aufgrund der Ruperts-Überlieferung) auf jeden Fall offensichtlich, dass in Murrhardt im Laufe des 8. Jahrhunderts bereits ein christlicher Kristallisationspunkt vorhanden war. Der kann unten im Tal an der Stelle des späteren Klosters gelegen sein. Die archäologischen Befunde erlauben eine Deutung in diesem Sinne durchaus. Er könnte sich ebenso gut oben auf dem Berg in und um die Marien- bzw. Walterichskirche befunden haben. Die Bezeichnung cellula dürfte den Sachverhalt jedenfalls recht gut treffen. $\mathrm{Ob}$ man diese cellula nun als Klostergründungsversuch bezeichnet oder nicht, ist letztlich ein fruchtloser Streit um Begriffe.

Im Übrigen sei darauf hingewiesen, dass auch die Gründung des Klosters Ellwangen 764 nur holperig in Gang kam. Erst eine zweite Gründung einige Jahre später brachte die Dinge wieder einigermaßen ins Lot. Die Weihe der Klosterkirche wird erst auf 773 angesetzt. Das gesamte Kloster habe sich auch bis zum Beginn des 9. Jahrhunderts im Tal (in valle) befunden und sei erst dann an seine heutige Stelle verlegt worden. Überhaupt finden sich solche steckengebliebene Klostergründungen dieser Zeit gar nicht so selten. ${ }^{13}$ Auch bei der Gründung des Klosters Hirsau - neben Ellwangen und Murrhardt eines der ältesten Klöster im Bereich Württembergs - wird erwogen, dass dessen Gründung um 765/768 recht stockend verlief und erst um 830 richtig in Gang, d.h. in benediktinische Formen kam. ${ }^{14}$ Ellwangen erhielt - auch das wäre eine Parallele zu Murrhardt - 814,

effluit in vallem) kann eigentlich nur mit dem heutigen Fatzenbrünnele identisch sein. Eine andere Quelle, auf die die Beschreibung passt, gibt es nicht. Das Wasser des Fatzenbrünneles gilt noch heute als hochwertig und mineralhaltig und wird von verschiedenen Leuten in Flaschen abgezapft. 13 Winfried Böhne: Zur frühmittelalterlichen Geschichte Ellwangens nach Fuldaer Quellen. In: Ellwangen 764-1964. Beiträge und Untersuchungen zur Zwölfhundert-Jahrfeier. Hg. i. A. der Stadt Ellwangen/Jagst von Viktor Burr. Ellwangen 1964, S. 73-106. Immo Eberl: Kloster Ellwangen im Umkreis seiner Gründer. In: Wolfgang Schmierer (Hg.): Aus südwestdeutscher Geschichte. Festschrift für Hans-Martin Maurer. Stuttgart 1994, S. 73-80; zusammenfassend: Hans Pfeifer: Ellwangen. In: Wolfgang Zimmermann / Nicole Priesching (Hg.): Württembergisches Klosterbuch. Klöster, Stifte und Ordensgemeinschaften von den Anfängen bis in die Gegenwart. Ostfildern 2003, S. 224-227; Susanne Arnold / Andreas Gut u. a.: Zwei Brüder, ein Kloster. Die Abtei Ellwangen zwischen Frömmigkeit und Politik; auf der Suche nach den alamannisch-fränkischen Wurzeln der Klostergründung von 764. Texte zur Ausstellung: Sonderausstellung, 26.7.2014-18.1.2015, Alamannenmuseum Ellwangen. (Schriften des Alamannenmuseums Ellwangen 2). Ellwangen 2014. Der neueste Forschungsstand bei Immo Eberl: Gründung und Frühzeit der Abtei Ellwangen. In: Ellwanger Jahrbuch 44 (2012/13), S. 29-52; vgl. auch: Joachim Wahl: Auf den Spuren von Hariolf und Erlolf - anthropologische Untersuchungen der Skelettreste aus dem Schrein der Basilika St. Vitus in Ellwangen. In: ebd., S. 13-28; Susanne Arnold: Die Ausgrabungen auf dem Marktplatz in Ellwangen - Einblick in die Klostergeschichte. In: ebd., S. 133-152.

14 Karl Schmid: St. Aurelius in Hirsau 830(?)-1049/75. Bemerkungen zur Traditionskritik und zur 
also fast gleichzeitig wie dieses, eine Urkunde Ludwigs des Frommen, und ebenso wie dieses unter Bezugnahme auf eine verlorene, in die 770er Jahre zu datierende Urkunde Karls des Großen. ${ }^{15}$ Man hätte also in Ellwangen, Murrhardt und Hirsau durchaus ähnliche Verläufe, in denen viel auf eine stockende Anlaufphase hindeutet, die dann erst später auf ein fest organisiertes - im Sinne Wagners „reformiertes“ - Kloster nach benediktinischen Regeln hinausläuft. ${ }^{16}$

Wenn man dann noch in die Überlegungen mit einbezieht, dass in den 760er Jahren nicht nur in Ellwangen und Hirsau einschlägige Gründungsaktivitäten anzunehmen sind, sondern dass in ebendieser Zeit auch in Esslingen und Herbrechtingen von Abt Fulrad, dem Kaplan Pippins und Karls des Großen, Zellen des Klosters St. Denis gegründet wurden, die keineswegs schon fertige Klöster waren, ${ }^{17}$ dann hätte man weitere analoge Beispiele. Die 777 erwähnte Fulradszelle in Hoppetenzell bei Stockach und die in einer gefälschten Urkunde von 782 erwähnte Fulradszelle in (Schwäbisch?) Gmünd wurden offenbar nie zum Kloster ausgebaut. ${ }^{18}$ All dies weist darauf hin, dass im Gebiet des heutigen Südwestdeutschland karolingernahe Familien zu einem frühen Zeitpunkt solche Zellen gründeten. Diese lebten offenbar nach schwankenden Regeln, und einige von ihnen - nicht alle - entwickelten sich im weiteren Verlauf des späten 8. oder des frühen 9. Jahrhunderts zu (benediktinischen) Klöstern weiter.

Gründerproblematik. In: Hirsau St. Peter und Paul 1091-1991. (Forschungen und Berichte der Archäologie des Mittelalters in Baden-Württemberg 10), Stuttgart 1991, Bd. 2, S. 11-43; Theodor Klüppel: Der heilige Aurelius. Ein Beitrag zur Verehrungsgeschichte des Hirsauer Klosterpatrons. In: ebd., S. 221-258; Stephan Molitor: Ut fertur sub Pippino rege... Zur karolingerzeitlichen Gründung Hirsaus. In: ebd., s. 45-54; zusammenfassend: Stephan Molitor: Hirsau. In: Zimmermann/ Priesching 2003 (wie Anm. 12), S. 279 ff.

15 Eberl 2012/13 (wie Anm. 13), S. 37 f.

16 Es sei allerdings darauf verwiesen, dass Murrhardt in der Liste der insgesamt 48 namentlich genannten Reichsklöster (der notitia de servitio monasteriorum) fehlt, die Ludwig der Fromme 818/19 anlegen ließ. Emile Lesne hat angenommen, dass nur diejenigen Klöster in diese Liste aufgenommen wurden, die bereits nach der benediktinischen Reform des Benedikt von Aniane lebten (Emile Lesne: Les ordonnances monastiques de Louis le Pieux et la Notitia de servitio monasteriorum. In: Revue d'histoire de l'Église de France 11 (1920), S. 161-175, 321-338, 449-493. Der Herausgeber der Notitia ist Lesne darin gefolgt (Petrus Becker (Hg.): Notitia de servitio monasteriorum. In: Corpus Consuetudinum Monasticarum 1. Siegburg 1963, S. 483-499, hier 487 f). Wenn die Annahme von Lesne zuträfe, wäre Murrhardt also 818/19 noch nicht im Sinne der Aachener Klosterreformen umgeformt gewesen, was im Widerspruch zu Wagners Annahme stünde, Murrhardt wäre als eine Art benediktinisches Reform- und Vorzeigekloster gegründet worden. Vgl. auch Eberl 2012/13 (wie Anm. 13), S. 38.

17 Zu Esslingen: Franz Quarthal: Die Geschichte der Fulradszelle in Esslingen nach der schriftlichen Überlieferung. In: Günter Fehring / Barbara Scholkmann (Hg.): Die Stadtkirche St. Dionysius in Esslingen am Neckar. Stuttgart 1995 (= Forschungen und Berichte der Archäologie des Mittelalters in Baden-Württemberg 13/1), S. 483-510; zusammenfassend: Franz Quarthal: Esslingen, Fulradszelle. In: Zimmermann/Priesching (wie Anm. 13), S. 233 f. Zu Herbrechtingen: Wolfgang Zimmermann: Herbrechtingen. In: ebd., S. $272 \mathrm{f}$.

18 Josef Fleckenstein: Fulrad. In: Lexikon des Mittelalters. Bd. 4. München, Zürich 1989, Sp. 1024 f. Johannes Thum: Hoppetenzell, ein Beitrag zur frühen Geschichte und zum Namen des Ortes. In: Hegau 34 (1989), S. 251-255. Wolfgang Zimmermann: Schwäbisch Gmünd Fulradszelle(?). In: Zimmermann/Priesching 2003 (wie Anm. 13), S. 440. 
Bei Fulrad finden sich übrigens Indizien, die in die Umgebung des Murrhardter bzw. Neustadter Abtes Walterich weisen. Zunächst einmal war Fulrad 750 zusammen mit dem ersten Würzburger Bischof Burkhard, dem Vorgänger des Megingoz, im Auftrag des karolingischen (Noch-)Hausmeiers Pippin in Rom, um dort die Absetzung des letzten Merowinger-Königs Childerich III. und die Königssalbung Pippins auch seitens des Papstes in die Wege zu leiten. Verbindungen Fulrads nach Würzburg sind also nachgewiesen. Sodann erwähnt Fulrad in seinem Testament von 777 unter seinen Familienmitgliedern eine Schwester namens Waldradana. ${ }^{19}$ Wenn man die damalige Form der Namensvererbung kennt, bei der einzelne Namensteile zu neuen Namen zusammengesetzt wurden, bei der aber diese Namensteile Hinweise auf Verwandtschaft sind, dann kann man sich zumindest vorstellen, dass der Name von Fulrads Schwester Waldradana auf Verwandtschaft zur Walterichs-Sippe hindeutet: „Wald-“ weist auf die Walterichs-Sippe, ,-rad“ ist eindeutig aus „Fulrad“ entnommen, ,,-ana“ ist eine Feminin-Endung.

Nach Wagner befinden sich in einer 1982 von mir edierten Murrhardter Traditionsnotiz - von Wagner als Summarium bezeichnet $-{ }^{20}$ Teile einer offenbar echten Urkunde Ludwigs des Frommen von 816 (nicht 817). ${ }^{21}$ Diese Urkunde von 816 nennt als Gründungsausstattung des Murrhardter Klosters ein Gebiet, dessen Grenzen beschrieben werden bis Fichtenberg (Viheberg), bis Kaisersbach (Kaisersbuoch), bis zu einem clivus aquarum (auf den noch einzugehen sein wird) und bis zum Fluss Rot (ad rivum quod Rota vocatur). Außerdem habe das Kloster die Kirche mit einem Hof in Oßweil (beneficio ecclesiae Ossweil et curtae in eadem villa), Erdmannhausen mit Kirche und Hof (Ercka[n]marshausen cum ecclesia et curia) sowie das Dörfchen Laufen am Kocher (villula Lauffen prope fluvium, quod Kochar vocatur) erhalten. Diese Besitzaufzählung stimmt nicht ganz mit der überein, die in der im 12. Jahrhundert verfälschten Gründungsurkunde enthalten ist. Dort wird als Gründungsdotation angegeben: Die drei Pfarreien Fichtenberg, Murrhardt und Sulzbach, die Burg Hunnenburg, die der Kaiser zur Beruhigung der Brüder habe abbrechen lassen und aus deren Steinen die Klosterkirche erbaut worden sei, die Pfarreien mit Höfen in Oßweil und Erdmannhausen sowie ein Gut in Laufen, außerdem 35 (genauer: 37) namentlich genannte Hörige. Wagner führt aus, dass die Unterschiede zwischen

19 Fulrad schenkt seinen Besitz an das Kloster St. Denis zu seinem eigenen Seelenheil und dem seiner Eltern Riculf und Ermengard, seiner Brüder Gaustbert und Bonifacius sowie seiner Schwester Waldradana (pro anime meae et genitore meo Riculfo et genitrice mea Ermengarde et germano meo Gaustberto et Bonifacio et sorore mea Uualdradane), vgl. Michael Tangl: Das Testament Fulrads von Saint-Denis. In: Neues Archiv der Gesellschaft für ältere deutsche Geschichtskunde 32 (1907), S. 167-217, hier 209. Vgl. zur Romreise auch Fleckenstein 1989 (wie Anm. 18). Der Namensteil Gaust, Gaus = Goz (auf S. 213 in der Schreibung Gausbertus) weist ins Umfeld des Würzburger Bischofs Megingoz. Der Begriff germanus könnte darauf hinweisen, dass es sich nur um einen Halbbruder handelte.

20 Fritz 1982 (wie Anm. 1), S. 121; dasselbe neu ediert bei Wagner 2001 (wie Anm. 2), S. 445.

21 Wagner 2001 (wie Anm. 2). 
der in der originalen Urkunde von 816 und in der aus dem 12. Jahrhundert stammenden Überarbeitung von 817 daher rühren, dass das Kloster im 12. Jahrhundert seine zwischenzeitlich entstandenen Pfarreien Fichtenberg und Sulzbach, von denen 816 noch nicht die Rede ist, juristisch gesichert bestätigt haben wollte. Ein solches „Nachbessern“ alter Dokumente ist im Mittelalter nicht selten. Wagner führt weiter aus, dass er die Entstehungszeit der nachgebesserten Urkunde auf die 1160er Jahre eingrenzt und als Verfasser dieses Dokuments entweder den am Hof von Friedrich Barbarossa tätigen Schreiber Rainald G oder Wortwin annimmt. Damit kann er die von mir 1982 gemachte Datierung zeitlich etwas präziser fassen und einen mutmaßlichen Schreiber namhaft machen.

Über den realen Kern solcher im 12. Jahrhundert entstandenen Fälschungen gehen die Urteile der Historiker weit auseinander. Während Wagner insgesamt eher skeptisch ist, konnten z. B. beim Kloster Fulda die Forschungen zu den Fälschungen des 12. Jahrhunderts in diesen Fälschungen in erheblichem Umfang ältere Substanz nachweisen. ${ }^{22}$

\section{Walterich zwischen Neustadt, Murrhardt, Rom, Worms - und Aachen, Reims und Lérins?}

Wagners Argumentation ist auf die Urkunden von 816/817 fixiert, wo er insbesondere zu 816 in der Tat auch über das bisher Gesagte hinaus neue Erkenntnisse liefert. Eine zentrale Personenidentität wird bei Wagner ebenfalls erwähnt, die im Zusammenhang mit den Anfängen des Klosters Murrhardt nicht ignoriert werden darf: Der Murrhardter Gründungsabt Walterich, der 816/817 bei der Entstehung des Klosters eine entscheidende Rolle spielte, war offenkundig derselbe, der 794-796 im Kloster Neustadt am Main Abt war. Diese Jahre werden in der Neustadter Überlieferung genannt. Dort war der als Bischof von Würzburg zurückgetretene Megingoz Abt gewesen, und dessen Schüler Walterich war, wie ausdrücklich überliefert ist, von dessen Anhängern (oder von dessen Sippenmitgliedern? de suis) zum neuen Abt gewählt worden. Da Wagner aber annimmt, Megingoz sei nicht erst 794, sondern schon 783 gestorben, müsste Walterich nicht von 794-796 Abt in Neustadt gewesen sein. Der Beginn seines Neustadter Abbatiats wäre dann schon 783 anzusetzen. Weshalb er sein Amt verlor, ist nicht eindeutig ersichtlich. Maßgeblich waren wohl Differenzen zu Karl dem GroBen. ${ }^{23}$ Wagner erwägt demnach eine Amtszeit Walterichs als Neustadter Abt von 783-796, ${ }^{24}$ stellt aber auch zur Diskussion, ob Walterich 785/786 am Aufstand des Grafen Hartrad gegen Karl den Großen beteiligt gewesen und dann abgesetzt

22 Dazu Böhne 1964 (wie Anm. 13), S. 77.

23 Vgl. die Details bei Fritz 1982 (wie Anm. 1), S. 22, 32 f.

24 Heinrich Wagner: Die Äbte des Klosters Neustadt am Main im Mittelalter. In: Würzburger Diözesan-Geschichtsblätter 46 (1984), S. 5-60, hier 11. 
worden sein könnte (also 786), was freilich mit den in Neustadt überlieferten Jahreszahlen nicht übereinstimmen würde. ${ }^{25}$

Übrigens hätte die von Wagner postulierte Frühdatierung von Walterichs Abbatiat in Neustadt am Main einige Folgen für die Einschätzung von Walterichs Lebensalter. Wenn Walterich tatsächlich schon 783 Abt in Neustadt geworden wäre und nicht erst 794, dann müsste er 783 in nicht mehr ganz jugendlichem Alter gestanden sein (mindestens 30 Jahre - das kanonische Mindestalter für das Abtsamt betrug 30 Jahre). Er wäre demnach um oder bald nach 750 geboren und wäre bei der von Wagner näher beschriebenen Rom-Mission $816^{26}$ ein Mann von mindestens etwa 60-65 Jahren gewesen. Es ist nicht völlig ausgeschlossen, dass ein solcher, für damalige Verhältnisse wirklich alter Mann noch die beschwerliche Reise über die Alpen auf sich genommen hat. Zu Walterichs Reisen im Jahre 816 wird noch einiges zu sagen sein. Bei einem Geburtsdatum um oder bald nach 750 dürfte Walterichs Todesdatum nicht - wie bisher angenommen - „um 830 “ liegen, sondern eher relativ nahe an den Jahren 816/817, in denen er sich noch konkret fassen lässt. ${ }^{27}$

Walterichs Rolle bei der Romreise wird von Wagner 2001 wesentlich präziser formuliert, als ich das 1982 wagte. Zunächst einmal datiert Wagner die Romreise auf 816, nicht auf 817. Walterich wäre laut Wagner angesichts des nahen Todes von Papst Leo III. († 12. Juni 816) zusammen mit einem zweiten Gesandten nach Rom geschickt worden, um dafür zu sorgen, dass eine dem Kaiser genehme Person zum neuen Papst gewählt würde. Das habe zwar nicht mehr geklappt, weil man angesichts der herannahenden kaiserlichen Gesandtschaft binnen Tagesfrist einen neuen Papst wählte - Stephan IV. Aber Walterich und seine Begleiter Paschalis und Johannes hätten es dann geschafft, Stephan IV. zu einer konzilianten Haltung gegenüber dem Kaiser zu bewegen, seien dann eilends über die Alpen zurück nach Worms gereist, wo sie um den 20. Juli 816 Ludwig den Frommen getroffen und ein Treffen mit Stephan IV. in Reims vereinbart hätten. Dann seien sie erneut nach Italien gereist, um den Papst zu informieren, der im Oktober 816 den Kaiser in Reims getroffen hätte. Stephan IV. jedenfalls hatte nicht lange Gelegenheit, sich seines Amtes zu erfreuen, denn er starb schon am 24. Januar 817, gefolgt vom neuen Papst Paschalis, in dem Wagner den gleichnamigen Begleiter des Walterich vermutet. ${ }^{28}$

25 Wagner 1984 (wie Anm. 24), S. 5-60, hier 10ff; auch ders. 2001 (wie Anm. 2), S. 423. In dem ebenfalls von Wagner verfassten Beitrag: Die Würzburger Bischöfe 741-842. In: Würzburger Diözesan-Geschichtsblätter 65 (2003), S. 17-44, hier insbesondere 22 f, weist Wagner ausführlich auf die verschiedenen, sich z. T. erheblich widersprechenden Quellen zum Todesjahr des Megingoz (783? 794?) hin und kommt zum Schluss, dass 783 am wahrscheinlichsten sei.

26 Wagner 2001 (wie Anm. 2), S. 436; vgl. dazu auch oben im gleich folgenden Text.

27 Überlegungen in diesem Sinne auch bei Wagner 1984 (wie Anm. 24), S. 12.

28 Wagner 2001 (wie Anm. 2), S. 434-437. Im Juli 816 hätte Walterich auch die wiederholt erwähnte Urkunde für das Kloster Murrhardt erhalten (ebd., S. 431). 
Man muss ja annehmen, dass der Murrhardter Klostergründer zusätzlich auch auf der kaiserlichen Reformsynode von Aachen im August 816 anwesend gewesen sein müsste, denn dort wurden die Weichen für benediktinische Reformen und Klostergründungen gestellt. Genau ein solches benediktinisches Reformkloster wurde in Murrhardt ja gegründet, ${ }^{29}$ und Walterich hätte irgendwann im Jahre 816 dann auch noch nach Murrhardt reisen müssen, um hier das Gründungsprojekt zu realisieren. Man hätte dann eine ambitionierte Reiseroute Walterichs: Im Juni 816 vom kaiserlichen Hof nach Rom, im Juli von dort nach Worms, dann wieder nach Rom, dann im Juli womöglich auch noch nach Reims, von dort im August nach Aachen und von Aachen schließlich nach Murrhardt. Inwieweit ein solch hektisches Hin- und Herreisen über die Alpen und wieder zurück und dann offenbar gleich nochmal über die Alpen und dann womöglich gleich noch nach Reims, Aachen und auch noch nach Murrhardt angesichts des fortgeschrittenen Alters von Walterich wahrscheinlich ist, sei dahingestellt.

Wagner entwickelt seine Gedanken rund um Walterichs Vita noch weiter. Er erwägt, Walterich sei nach seiner Absetzung in Neustadt (786? 796?) nach Lérins an der Côte d'Azur in der Nähe von Cannes verbannt worden. Eine nicht eindeutig lesbare Formulierung in der Urkunde von 816 ist für ihn Anlass anzunehmen, der darin erwähnte „Berg nahe unserem Kloster“ sei der Berg in Lérins gewesen, „auf dem im 11./12. Jahrhundert [...] die Klosterfestung St.-Honoré-de-Lérins erbaut wurde“. In Lérins lag im 8. Jahrhundert das Kloster auf einer Insel (von der freilich in der Urkunde mit keinem Wort die Rede ist) und oberhalb der Insel gab es den erwähnten Berg, auf dem nach Wagner Walterich gelebt haben sollte. Ja, Wagner scheint implizit geradezu in Abrede zu stellen, dass Walterich - wie es die Urkunde von 817 und die örtliche Tradition will - als Einsiedler (heremita) in Murrhardt gelebt habe. ${ }^{30}$ Dass Walterich kein weltabgewandter Einsiedler und keineswegs eine Art Waldschrat gewesen ist, sondern der bedeutende Angehörige einer weiträumig handelnden und mit den Karolingern möglicherweise verwandtschaftlich verflochtenen Adelssippe (auf die Wagner überhaupt nicht eingeht), habe ich 1982 ausführlich dargetan. ${ }^{31}$ Aber Wagner fügt mit seiner These vom Exil Walterichs in Lérins eine völlig neue Komponente hinzu.

29 Murrhardt als „eine Art karolingisches Musterkloster“ auch bei Wagner 2001 (wie Anm. 2), S. 428 und ders. 1984 (wie Anm. 24), S. 11.

30 Wagner 2001 (wie Anm. 2), S. 423; auf S. 445 liest er: Walterich qui in monte prope monasterium nostrum ereenum conaspi[ra]verat [...] und meint, ereenum sei eine Verschreibung für lirinum $=$ Lérins. Angesichts der flüchtigen Handschrift und der offenkundigen Verschreibung dieser Stelle ist dies eine gewagte Interpretation und eine kühne Verbannungstheorie. Vgl. zum Aufstand Hardrads nach Einhards Vita Karoli Magni: Karl Brunner: Oppositionelle Gruppen im Karolingerreich. Wien, Köln, Graz 1979 (= Veröffentlichungen des Instituts für Österreichische Geschichtsforschung 25), S. 49ff; knapp auch: Rudolf Schieffer: Die Karolinger. (Urban-Taschenbücher 411), Stuttgart 1992, S. 83.

31 Fritz 1982 (wie Anm. 1), S. 19-37. Vgl. zu den diversen Waltrichen und ihren weitgespannten Beziehungen zu Ellwangen auch Hansmartin Schwarzmaier: Sozialgeschichtliche Untersuchungen zur Geschichte der Abtei Ellwangen in der Karolingerzeit. In: Burr 1964 (wie Anm. 13), S. 50-72, insbesondere 61-64. 
Zur Lérins-Deutung ist zu sagen: Da es in Murrhardt einen Berg direkt neben dem Kloster des Kaisers gibt, muss man nicht bis an die Côte d'Azur gehen, um eine Kloster-Berg-Konstellation zu finden. Warum wohl wäre denn Walterich nach seinem Tod auf dem Berg nahe dem Kloster Murrhardt (in der damaligen Marienkirche, der heutigen Walterichskirche) begraben worden? Man könnte daran denken, dass bei seinem Tod das Kloster erst im Bau war, sodass dort seine Bestattung nicht möglich war. Aber das leuchtet nicht unbedingt ein: Man hätte Walterich ohne weiteres auch in einer noch im Bau befindlichen Klosterkirche oder in dem vor Walterichs Zeiten erbauten (sakralen?) Gebäude östlich der späteren karolingischen Klosterkirche begraben können. Viel einleuchtender ist ein anderer Grund für sein Begräbnis oben auf dem Berg: Man legte wohl einfach Wert darauf, ihn dort zu begraben, wo er gelebt und gewirkt hatte. Allein schon die Tatsache, dass man hier für die Steinplatten zu seinem Grab eine aus dem 2./3. Jahrhundert stammende Romulus- und Remus-Skulptur verwendete, ${ }^{32}$ um den Triumph des Christentums über das Heidentum zu symbolisieren, unterstreicht die Bedeutung des Ortes und Walterichs hiesige Tätigkeit.

Wenn man schon eine politisch bedingte Verbannung Walterichs durch Karl den Großen annehmen will (und dafür spricht eigentlich alles), dann muss man nicht an das weit entfernte Lérins denken. In einem zeitlich nahe an Walterich liegenden Vergleichsfall liegt ebenfalls eine Verbannung an einen geographisch recht nahe befindlichen Ort vor: Otmar († 759), der zweite Abt des Klosters St. Gallen, wurde, nachdem er in Ungnade gefallen war, auf das Inselchen Werd bei Stein am Rhein verbannt - gerade etwa $30 \mathrm{~km}$ Luftlinie von St. Gallen entfernt. ${ }^{33}$ Eine Verbannung Walterichs wäre auch durchaus nach Murrhardt möglich gewesen, das im Übrigen mit 105 km Luftlinie deutlich weiter von Neustadt entfernt ist als Werd von St. Gallen.

Im Falle Walterichs sind einige Informationen der Urkunde von $817 \mathrm{zu}$ beachten: In dieser wird Merkwürdiges über die - weiter unten noch geographisch und topographisch näher zu beschreibende - „Hunnenburg“ berichtet: Neben dieser dem Kaiser gehörenden Anlage habe Walterich mit Erlaubnis Ludwigs des Frommen im Walde gelebt (in nemore prope castrum nostre proprietatis, quod vulgo Hunemburg dicitur) und sei, wie eingangs beschrieben, von Ludwig sogar zum Beichtvater gewählt worden. Nicht lange danach (also wohl, nachdem Walterich zum Beichtvater erwählt worden war) habe der Kaiser ihm die zu Beginn des vorliegenden Beitrags erwähnten zwölf Brüder hinzugegeben. Diese sollten Walterichs Einsiedlerleben umwandeln, damit mönchisches Leben nach der Regel des heiligen Benedikt geführt werde. Und nun kommt das Verblüffende: Die

32 Vgl. die Abbildung bei Bodo Cichy: Murrhardt. Sagen, Steine, Geschichten. Murrhardt 1963, S. 31 .

33 Johannes Duft: Die Lebensgeschichten der Heiligen Gallus und Otmar, aus den lateinischen Viten übersetzt und herausgegeben. (Bibliotheca Sangallensis 9). St. Gallen, Sigmaringen 1988; ders.: Der Zönobit Sankt Otmar (†759). Die Quellen zum Otmars-Leben. In: ders.: Die Abtei St. Gallen. Bd. 2: Beiträge zur Kenntnis ihrer Persönlichkeiten. Sigmaringen 1991, S. 39-49. 
Mönche fühlten sich offenbar von der Hunnenburg beunruhigt, und deshalb habe der Kaiser angeordnet, dieselbe $\mathrm{zu}$ deren Beruhigung zu zerstören (castrum Hunnemburg pro quiete fratrum destrui). Normalerweise bot eine kaiserliche Burg Schutz. Weshalb wohl sollten sich die Mönche von einer solchen Burg beunruhigt fühlen? Nun, wenn Walterich tatsächlich von Karl dem Großen nach Murrhardt ins Exil geschickt wurde, dann musste er dort kontrolliert werden und er konnte natürlich bestens durch die Hunnenburg und deren Besatzung kontrolliert werden, während er, politisch kaltgestellt, ,im Walde“ notgedrungen als Einsiedler lebte. Dass er da im Laufe der Zeit keine Sympathien für die nahe gelegene Burg entwickelt hat, ist leicht nachvollziehbar.

\section{Zur Geographie und Topographie der frühen Quellen}

Zweifellos getäuscht hat sich Wagner mit der 817 erwähnten Zuordnung von Sulzbach. Es gibt keinerlei Hinweis, dass damit - wie Wagner schreibt - Sulzbach am Kocher gemeint sein könnte. Dort war Murrhardt, soweit ersichtlich, niemals begütert. ${ }^{34}$ Vielmehr handelt es sich eindeutig um Sulzbach an der Murr, das auch in den folgenden Jahrhunderten bis in die frühe Neuzeit eng mit Murrhardt verflochten war.

Auch weitere Ortsidentifikationen von Wagner sind näher zu betrachten. Das 817 erwähnte Vrankenuurt ist nach aller Wahrscheinlichkeit keine Furt an der Murr. Die Murr ist noch bis viele Kilometer unterhalb Murrhardts ein derart bescheidenes Gewässer, dass eine Furt zu ihrer Überquerung eigentlich nicht erforderlich ist. Außerdem gibt es nirgendwo den geringsten Hinweis auf einen in der Nähe Murrhardts befindlichen Orts- oder auch nur Flurnamen, der nur entfernt an eine an der Murr gelegene „Frankenfurt“" erinnern würde - zumal an diese „Frankenfurt“ ja eine Ansiedlung von nicht weniger als 25 Männern gewesen sein müsste (zu denen deren Familien zu zählen wären, also insgesamt wohl über 100 Personen). ${ }^{35}$ „Frankenfurt“ bleibt, wie ich es 1982 genannt habe, nicht identifizierbar. ${ }^{36}$ Ganz unklar ist, weshalb in der gefälschten Urkunde von 817, die ja nach Ansicht der damit befassten Historiker den Zweck hatte, Murrhardter Besitztitel zu untermauern, derart ausführlich auf das in dieser Hinsicht völlig sinnlose, weil nirgends lokalisierbare „Frankenfurt“ eingegangen wird.

Clivus aquarum mit vorderem und hinterem Wasserfall (am Hörschbach südwestlich Murrhardt) zu identifizieren, wie Wagner es tut, ist nicht haltbar. Wasserfälle gibt es - wie u. a. die Standorte späterer Säg- und Ölmühlen an verschie-

34 Zur Identifikation Sulzbachs mit Sulzbach am Kocher Wagner 2016 (wie Anm. 5), S. 60 . Vgl. Gerhard Fritz: Stadt und Kloster Murrhardt im Spätmittelalter und in der Reformationszeit. Sigmaringen 1990 (=FWFr 34), S. 235.

35 WUB 1, Nr. 78, S. 87 ff: Vgl. die „Frankenfurt“ zugeordneten Namen unten Anm. 40. Dazu auch Wagner 2001 (wie Anm. 2), S. $433 \mathrm{f}$.

36 Fritz 1982 (wie Anm. 1), S. 53 f. 
denen Sandsteinstufen zeigen - rund um Murrhardt mehrere. Zunächst einmal erschließt sich nicht, weshalb gerade der in unmittelbarer Klosternähe gelegene vordere bzw. hintere Wasserfall gemeint sein soll. Beide sind zudem in dem heute noch als ,wildromantisch“ bezeichneten engen Hörschbachtal schwer zugänglich und dürften im 9. Jahrhundert als Wegmarken kaum erreichbar und im Dickicht des Waldes kaum sichtbar gewesen sein. Die von Wagner neu edierte Urkunde spricht von einem Murrhardter Gründungsbesitz eine Meile weit in vier Richtungen (datis terminis ab ipsa fundatione in 4 partes milliariorum), nämlich bis Viheberg, dann Kaisersbuoch, schließlich bis zum clivus aquarum und bis zum Fluss Rota. Alle identifizierbaren Grenzmarkierungen sind weit vom Kloster entfernt, nämlich in der Größenordnung einer (deutschen) Meile (ca. 7 $\mathrm{km}$ ): Fichtenberg etwa $10 \mathrm{~km}$ Luftlinie, die Rot auf einer ganzen Strecke mindestens etwa 7-8 km, ebenso Kaisersbach, die beiden von Wagner genannten Wasserfälle (clivus aquarum) dagegen gerade einmal $2 \mathrm{~km}$. Das erscheint ganz unwahrscheinlich. Angesichts der Lage der anderen Grenzlokalisierungen ist es klar, dass die Stelle mit dem clivus aquarum sich auf die südliche Grenze der Murrhardter Gründungsdotation bezieht.

Man dürfte der Wahrheit etwas näher kommen, wenn man die berühmte Urkunde von 1027 heranzieht, die den damaligen Wildbannbezirk des Klosters Murrhardt beschreibt. ${ }^{37}$ Dort ist die Rede davon, dass die Grenze dieses Bezirks sich von der Quelle der Wieslauf bis nach Sechselberg und von dort mitten zwischen dem Hörschbach und der Weissach bis zum Eichelberg (bei Unterbrüden) erstrecke (ab ortu rivi qui dicitur Wisilaffa usque ad montem Sassenberch et sic per medium duorum rivorum Heroltosbach et Wizzahe et ita ad montem Eichenesberch). Von Südosten her - dort wo 816 von Kaisersbuoch die Rede ist - wird 1027 die Wildbanngrenze nur summarisch beschrieben: Sie gehe vom Steigersbach aus, der etwa von Gschwend aus in den Kocher fließt, die Grenze zwischen den Franken und den Schwaben entlang bis zur Quelle der Wieslauf (Steigirisbach et sic per confinia Francorum et Sueuorum usque ad supra dicta fontem Wisilaffa). 1027 wird also mit der Mitte zwischen Hörschbach und Weissach und mit dem Höhenzug vom Steigersbach zur Wieslaufquelle wiederholte Male eine Wasserscheide beschrieben. Genau das dürfte auch 816 gemeint sein: Der Begriff clivus heißt zunächst einmal nur Abhang, clivus aquarum dürfte demnach nicht als „Wasserfall“ zu verstehen sein, sondern als Wasserscheide. Mit dieser Deutung läge man, was die Südgrenze der Gründungsdotation von 816 angeht, in derselben Entfernung von Murrhardt wie bei den anderen 816 genannten Grenzmarkierungen und nicht bei den beiden Hörschbachwasserfällen, die ja, wie erwähnt, in unmittelbarer Nähe Murrhardts liegen. 
Die bisherige Literatur ist überhaupt noch nicht auf die Nennung von Kaisersbuoch in der Urkunde von 816 eingegangen. Das verwundert, da die Nennung der anderen Örtlichkeiten - zum Nachweis für dieses oder jenes - immer wieder behandelt wurde. Von der Namensform Kaisersbuoch und von der geographischen Lage her (im Südosten des Klosters) ist die Identifikation mit dem heutigen Kaisersbach unstrittig, zumal für Kaisersbach die Namensform Kaiserspůch noch 1375, 1379 und 1382 überliefert ist. Erst 1448 ist dann von Kaiserspach die Rede. ${ }^{38}$ Seltsamerweise geht auch keine der gefälschten Urkunden von 788 und 817 auf Kaisersbach ein, und auch in der Wildbannurkunde von 1027 spielt es keine Rolle. Die Nichtnennung Kaisersbachs in all diesen Urkunden, in denen es ja um die Rechtfertigung klösterlicher Eigentums- bzw. Besitzrechte geht, ist wohl nur damit zu erklären, dass das Kloster in Kaisersbach weder 1027 noch im 12. Jahrhundert, als die Urkunde von 817 in der heutigen Form verfasst wurde, eben irgendwelche Rechte und irgendwelche Rechtsansprüche (mehr?) hatte und geltend machte. Erstaunlich ist dies durchaus, da die Formulierung der Urkunde von 816 eine Rechtsgrundlage für spätere Forderungen hätte bieten können.

Weiterhin ist hier nochmals auf die bereits oben erwähnte Hunnenburg zurückzukommen. Die in der Urkunde von 817 beschriebene Zerstörung der kaiserlichen Hunnenburg „,zur Beruhigung der Brüder“, also der Mönche, in der Nähe des Klosters ist schwerlich eine bloße Erfindung der Fälscher des 12. Jahrhunderts. Wie ich 1982 gezeigt habe, handelt es sich bei der Hunnenburg keineswegs um jene kleine, hochmittelalterliche Anlage oberhalb der Eisenschmiedmühle und der Wahlenmühle zwischen Murrhardt und Fornsbach, ${ }^{39}$ sondern um die Ruinen des römischen Kastells. Die erst in später Überlieferung als Hunnenburg bezeichnete Anlage oberhalb der Wahlenmühle ist etwa $4 \mathrm{~km}$ vom Kloster entfernt und dürfte wegen ihrer Kleinheit nie eine Bedrohung für dieses gewesen sein. Außerdem passt die Wahlenmühle-Hunnenburg schon aus chronologischen Gründen nicht zum 12. Jahrhundert. Dass gerade diese Anlage im Mittelalter je als Hunnenburg bezeichnet wurde, ist außerdem nirgends gesichert und eher unwahrscheinlich. Der Berg, auf dem die heute völlig verschwundene Anlage lag, wird in den einschlägigen Karten nur mit Flurnamen wie „Burgberg“, „Burgreute“, „Kasten“ und „Burstel“ (für Burgstall) bezeichnet, nie mit „Hunnenburg“. Demgegenüber lag das römische Kastell, das man sich - unklar über seine tatsächlichen Ursprünge - als von den Hunnen erbaute Burg erklärte, tatsächlich in unmittelbarer Nähe des Klosters, und tatsächlich wurden - wie archäologisch nachgewiesen werden konnte - seine Steine zum Bau der Klosterkirche verwen-

38 Lutz Reichardt: Ortsnamenbuch des Rems-Murr-Kreises. (Veröffentlichungen der Kommission für geschichtliche Landeskunde in Baden-Württemberg, Reihe B, 128). Stuttgart 1993, S. $174 \mathrm{f}$.

39 Vgl. zu dieser Anlage den Artikel von Gerhard Fritz: Murrhardt, Hunnenburg bzw. Burgberg. In: Gerhard Fritz, Roland Schurig (Hg.): Die Burgen im Rems-Murr-Kreis. Remshalden 1994, S. 85 f. Dazu und zu den möglichen Bewohnern der Wahlenmühle-Hunnenburg Gerhard Fritz: Fornsbach bis um 1800. Künftig in: Jubiläumsbuch Fornsbach. 
det, genau wie das die Urkunde von 817 beschreibt: Aus der Hunnemburg sei mit deren Steinen die Kirche gebaut worden (eisdem lapidibus ecclesiam aedificari).

\section{Die 817 genannten Personen}

Ein in der bisherigen Forschung wenig beachtetes Thema sind die angeblich 35 - bei genauer Zählung dann sogar 37 - Namen von Personen, die in der Urkunde von 817 auftauchen und die als Zubehör der verschiedenen Orte dem Kloster bei seiner Gründung übergeben worden seien. ${ }^{40}$ Allgemein geht man davon aus, dass es sich bei diesen Namen um Zutaten der Fälscher des 12. Jahrhunderts handelt. Insbesondere die im Zusammenhang mit den Personen z. T. verwendete Bezeichnung ministeriales und milites ist für das 9. Jahrhundert nicht denkbar, sehr wohl allerdings die ebenfalls verwendete Bezeichnung mancipia. Ministeriales und milites (,Dienstmannen und Ritter“) gehören eindeutig der Gesellschaft und der Terminologie des 12. Jahrhunderts an, mancipia sind dagegen im 9. Jahrhundert eine gängige Bezeichnung für Hörige, die zu einem Anwesen gehören. ${ }^{41}$

Etliche der Namen sind Allerweltsnamen, die ohne Weiteres ins 12. Jahrhundert passen, in dem die Urkunde von 817 verfasst wurde (z. B. Gerardus, Hartmanus, Adolfus, Ludevicus, Giselbertus, Heynricus). ${ }^{42}$ In diesen Fällen könnte in der Tat die wenig schöpferische Phantasie des Fälschers Pate gestanden haben - auch wenn mehrere dieser Namen (Ludwig!) durchaus auch für das 9. Jahrhundert denkbar sind. Unter den 37 Namen sind aber etliche, die offenkundig nicht ins 12. Jahrhundert, sondern ins Frühmittelalter passen (Gisolfus, Waldemer, Willekind, Nanthot, Waltmundt, Sintherus, Haddo, Regilo, Enhilo, Hemmo, Rubboldus). Insbesondere Waldemer, Willekind, Nanthot, Waltmund und Sinther sind - wie eine EDV-Überprüfung des Württembergischen Urkundenbuches $\operatorname{ergab}^{43}$ - eindeutig frühmittelalterliche Namen. Einige kommen überhaupt nur in der Urkunde von 817 vor.

40 WUB 1, Nr. 78, S. 87ff: De castro Hunnenburg Gisolfus et Richerus fratres, et quinque filii ipsorum, Gisolfus et Brungerus, Richerus, Wicmarus et Rumarus, cum aliis quatuor strenuis eiusdem castri militibus: Diethone, Waldemero, Egilone et Willekindo, nec non omni familia earum. De Vrankenuurt: Ludewicus, Adelgerus, Richerus, Wicmarus, Ruothardus et Wortwinus, Nanthot, Waltmundt, Sintherus, Haddo, Rubboldus, Richerus, Adolfus, Hiltebertus, Regilo, Adelramus, Gerardus, Hartmanus, Enhilo, Hemmo, Gotebald, Ludevicus et fratres sui duo Wimarus et Wicmarus, cum nepotis ipsorum Giselberto et Heynrico.

41 Dazu Fritz 1982 (wie Anm. 1), S. 53; Wagner 2001 (wie Anm. 2), S. 433 f.

42 Heinrich als Übernahme eines typischen Königs- bzw. Kaisernamens erscheint in Deutschland erst im Hochmittalter; vgl. Michael Mitterauer: Ahnen und Heilige. Namengebung in der europäischen Geschichte. München 1993, S. 275.

43 Gisolf: ein Beleg (nur 817), Waldemer (dto.), Willekind (dto.), Nanthot (dto.), Waltmundt (dto.), Sinther (dto.), Haddo (3 Belege), Regilo (2 Belege, der zweite von 1154), Enhilo (ein Beleg, nur 817), Hemmo (3 Belege, 817, 861, 1091, letzterer der Mainzer Erzbischof des 11. Jahrhunderts), 
Nun kann man natürlich annehmen, dass der Fälscher des 12. Jahrhunderts einfach ein kluger, raffinierter Kopf war, der seine Namenslisten archaisierend zusammenkomponieren konnte. Dagegen spricht freilich, dass die Zielgruppen des Fälschers - die Murrhardter Mönche oder übergeordnete Kanzleien - im 12. Jahrhundert kaum sprach- und namensgeschichtliche Feinheiten beurteilen konnten und wohl auch mit damals modernen Namen zufrieden gewesen wären. Anders gesagt: Es spricht einiges dafür, dass die archaischen Namen unter den Manzipien der Urkunde von 817 keine bloße Phantasie des Fälschers waren. Es ist denkbar, dass im Kloster Murrhardt tatsächlich irgendwelche Namenslisten des frühen Mittelalters aufbewahrt wurden, die dem Fälscher als Vorlage gedient haben könnten. Vielleicht waren sie auch Teil der von Wagner beschriebenen Kaiserurkunde von 816. In anderen Urkunden des 9. Jahrhunderts - so z. B. in der Gründungsurkunde des Klosters Wiesensteig von 861 - sind sehr wohl Listen von Namen der zugehörigen Manzipien enthalten, sodass man sich vorstellen kann, dass die originale Vorlage für den Fälscher - also wohl die von Wagner erkannte, nur verkürzt überlieferte Kaiserurkunde von 816 - solche Namen enthielt. ${ }^{44}$

\section{Fazit}

Ausgehend von meiner eigenen, 1982 erschienenen Untersuchung und verschiedenen, 1984, 2001 und 2003 veröffentlichten Beiträgen Heinrich Wagners wurde die Frühgeschichte des Klosters Murrhardt erneut aufgerollt. Noch einmal konnte betont werden, dass die Urkunden von 788 und 817 Fälschungen bzw. Überarbeitungen sind. Sie sind wohl um 990 bzw. in den 1160er Jahren entstanden. Wagners These, dass sich in den seinerzeit von mir und dann erneut von ihm selbst herausgegebenen Traditiones Murrhardtenses (bei Wagner: Summarium) die Formulierungen aus einer verlorengegangenen Urkunde Ludwigs des Frommen von 816 befinden, ist durchaus einleuchtend. Demnach hätte die Gründungsdotation Murrhardts 816 vom Kloster aus bis zur Rot, nach Fichtenberg, Kaisersbach und bis zur Wasserscheide südlich von Murrhardt gereicht. Außerdem hätten Oßweil, Erdmannhausen und Laufen am Kocher zur Gründungsdotation gehört. Die Pfarreien Murrhardt, Sulzbach und Fichtenberg, die in der Fälschung von 817 erwähnt werden, seien dagegen erst später von Murrhardt aus gegründet worden.

Die Details Wagners zur Vita des Murrhardter Gründungsabts Walterich bleiben dagegen zumindest teilweise unsicher: 783 (794?) Abt in Neustadt am Main als

Rubboldus (ein Beleg, nur 817). Vgl. zu diesen Namenstypen Mitterauer (wie Anm. 42), S. 229240.

44 WUB 1, Nr. 136, S. 159 ff. Wagner 2001 (wie Anm. 2), S. 433 f meint dagegen, die Namensliste sei separat aufbewahrt worden und nicht Teil der Gründungsurkunde gewesen. 
Nachfolger des Megingoz. 786 (oder 796?) von dort auf Betreiben Karls des Großen weggebracht (ob wegen der Beteiligung am Aufstand des Grafen Hartrad 785/86?). Ein von Wagner erwogener Aufenthalt Walterichs in Lérins als lectio difficilior wirkt weit hergeholt. Dessen Exil in Murrhardt ist, wie im Einzelnen dargestellt wird, wesentlich plausibler.

Walterichs politische Aktivitäten unter Ludwig dem Frommen waren zweifellos erheblich und dürften durchaus nach Rom geführt haben. Es wäre reizvoll anzunehmen, dass Walterich das Treffen zwischen dem Kaiser und dem Papst in Reims 816 eingefädelt hat. Wenn der damals aber nach Wagners Frühdatierung schon ziemlich alte Walterich binnen weniger Monate tatsächlich ständig und mehrfach zwischen Rom, Worms, Murrhardt und womöglich auch noch nach Aachen und Reims hin- und hergereist wäre, würde das eine fast nicht vorstellbar robuste Konstitution voraussetzen. Sollte Walterich 816 tatsächlich so extrem viel gereist sein, würde man das eher einem etwas jüngeren Mann zutrauen. D. h.: Entweder war Walterich 816 tatsächlich ein Mann von 60 und womöglich deutlich mehr Jahren, dann wären seine vielen Reisen eher unwahrscheinlich; oder er reiste 816 so viel wie beschrieben, dann hätte er aber eher jünger sein müssen und wäre dann nicht schon 783 auf Megingoz als Abt von Neustadt gefolgt. Hier muss letztlich Manches unsicher bleiben.

Unsicher bleiben auch verschiedene Überlegungen Wagners zur Identifikation mehrerer 816 genannter Orte. Dagegen ist es durchaus vorstellbar, dass verschiedene der in der Urkunde von 817 als Zubehör der Murrhardter Gründungsausstattung genannten Männer (Manzipien) tatsächlich in der verlorenen echten Urkunde (von 816?) genannt waren.

Die Existenz christlicher Aktivitäten in Murrhardt lange vor Walterich - einer cellula, wie die gefälschte Urkunde von 788 wohl nicht unrichtig angibt - dürfte dagegen gesichert sein, auch wenn Wagner das Vorhandensein eines Klosters oder auch nur eines Klostergründungsversuchs ausschließt. Archäologische Befunde, chronikalische Mitteilungen und naheliegende Plausibilitäten sprechen da eine eindeutige Sprache. Ebenso wurden die Mitteilungen der gefälschten Urkunde von 817 über den Bau der Klosterkirche aus den Steinen des als „Hunnenburg“ bezeichneten römischen Kastells archäologisch bestätigt. Die Frage, ob der 816/17 durchgeführten benediktinischen Klostergründung ein mehr oder weniger gescheiterter Klostergründungsversuch vorausging, lässt sich zwar nicht endgültig entscheiden, es weisen aber zusätzlich zu den archäologischen Funden mehrere parallel gelagerte Fälle (z. B. Ellwangen, Hirsau, evtl. Esslingen, Herbrechtingen, [Schwäbisch?] Gmünd, Hoppetenzell) darauf hin, dass eine solche Möglichkeit durchaus einiges an Substanz hat. Zu beachten ist nicht nur der Reichenauer Hinweis auf Pirmins Aktivitäten in Murrhardt, der in die Zeit um 750 weist. Der bislang völlig unbekannt gebliebene Hinweis auf den heiligen Rupert in Murrhardt deutet schemenhaft sogar noch um einiges weiter in die Vergangenheit, auch wenn sich konkrete Aussagen verbieten. 


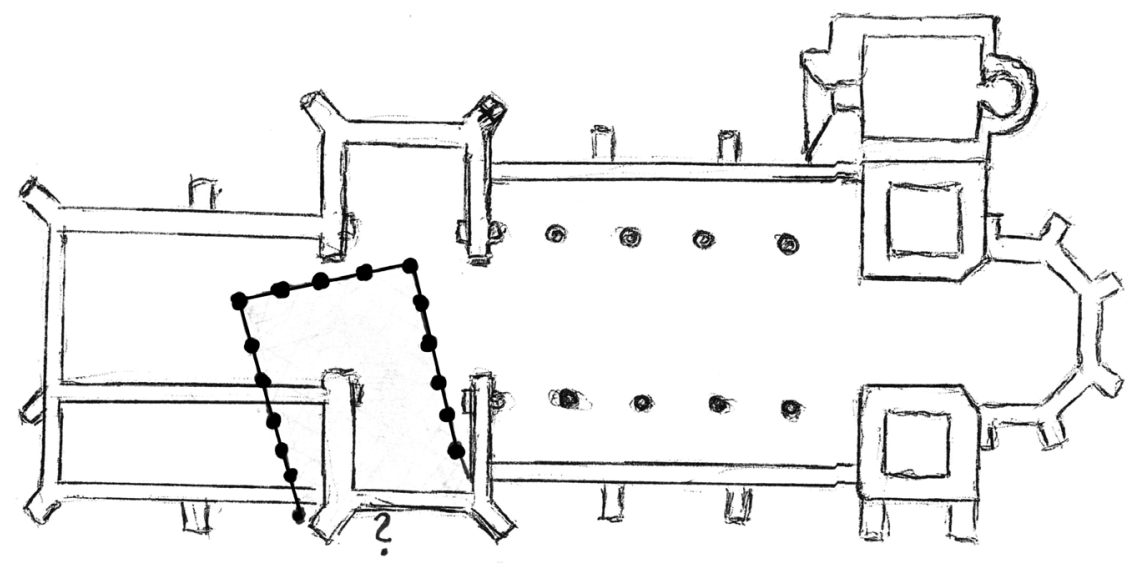

Römischer Holzbau ca. 150 - 200 n.Chr.

Abb. 1 Römischer Holzbau, ca. 150/200

(Alle Bilder: Carl-Schweizer-Museum Murrhardt).

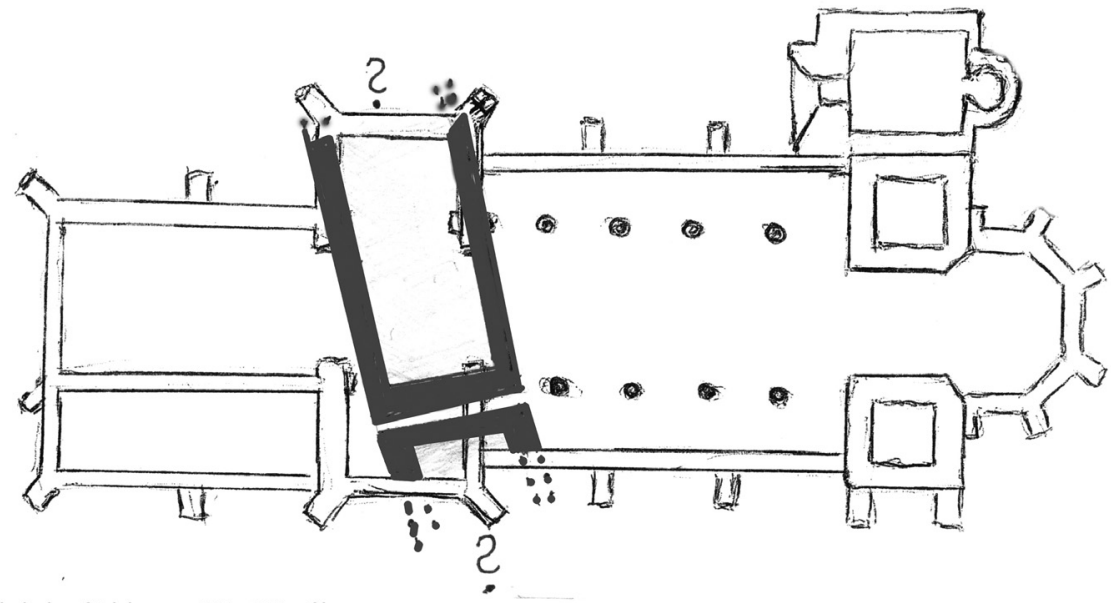

Römischer Steinbau ca. 200 - 260 n.Chr

Abb. 2 Römischer Steinbau, ca. 200-260. 


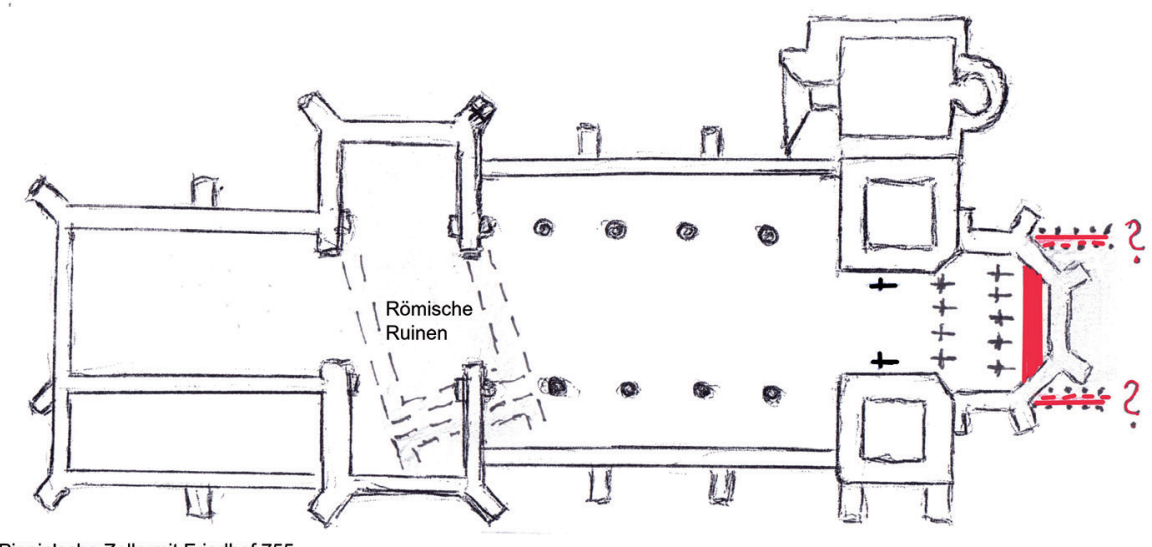

Pippin'sche Zelle mit Friedhof 755 St.Trinitatis

Abb. 3 Merowingischer bzw. frühkarolingischer Bau (Kirche?) am Ostende der heutigen Kirche.

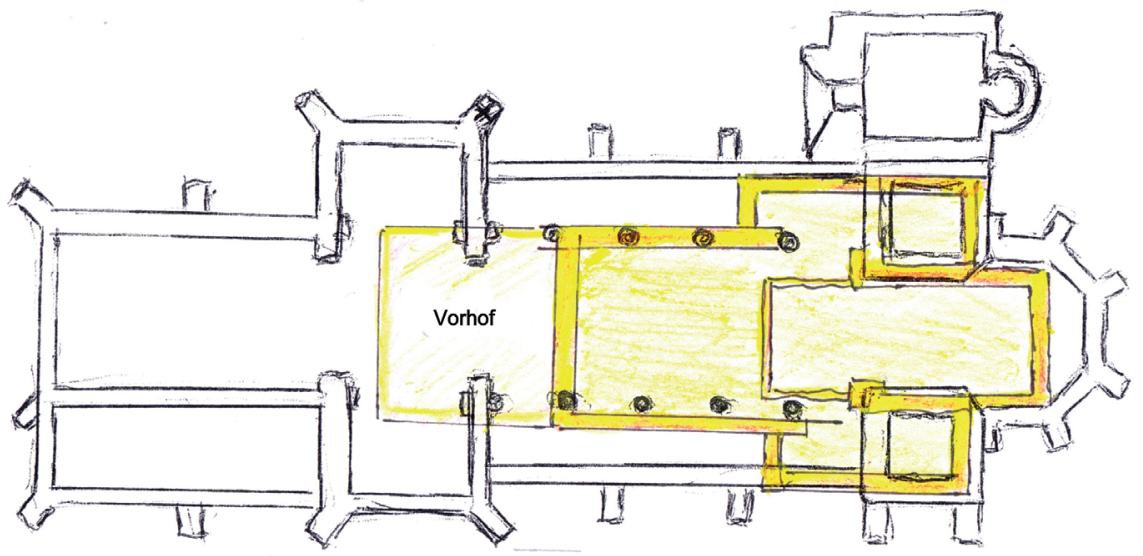

Karolingische Klosterkirche ca. 820

St. Trinitatis ; St. Johannes der Täufer ; St. Maria

Abb. 4 Karolingische Klosterkirche ca. 820, St. Trinitatis, Johannes der Täufer, Maria. 


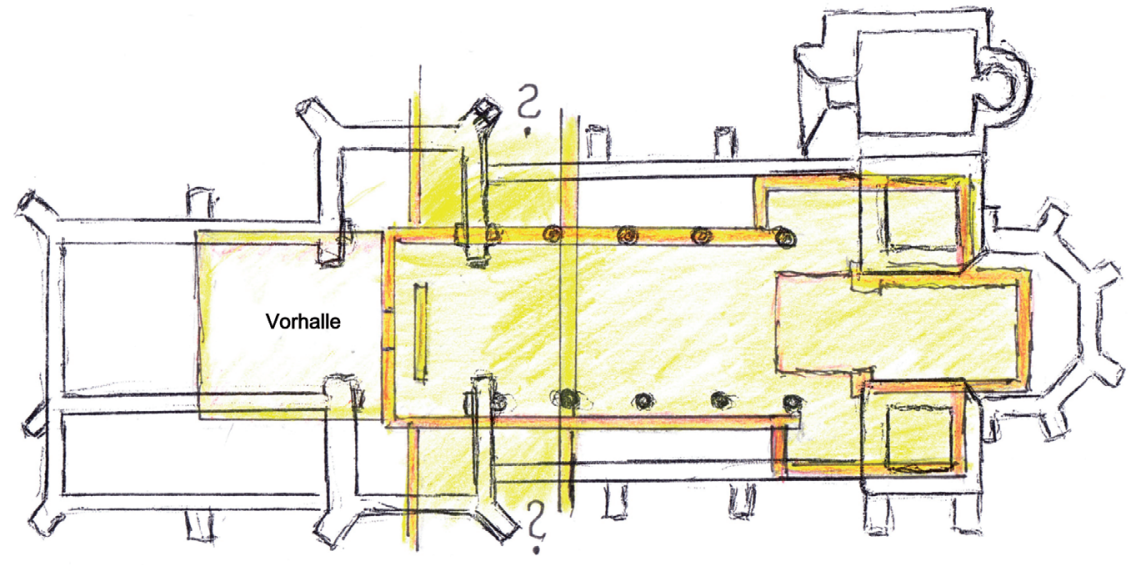

Erweiterte Klosterkirche St. Januarius ca. 840

Abb. 5 Erweiterte Kirche ca. 840, St. Januarius (nach der Translation der Januarius-Reliquien von der Reichenau, frühestens 838). 